PROCEEDINGS OF THE

AMERICAN MATHEMATICAL SOCIETY

Volume 136, Number 6, June 2008, Pages 2055-2065

S 0002-9939(08)09342-8

Article electronically published on February 20, 2008

\title{
BIFURCATION OF HOMOCLINICS OF HAMILTONIAN SYSTEMS
}

\author{
JACOBO PEJSACHOWICZ \\ (Communicated by Carmen C. Chicone)
}

\begin{abstract}
We obtain sufficient conditions for bifurcation of homoclinic trajectories of nonautonomous Hamiltonian vector fields parametrized by a circle, together with estimates for the number of bifurcation points in terms of the Maslov index of the asymptotic stable and unstable bundles of the linearization at the stationary branch.
\end{abstract}

\section{INTRODUCTION}

In [6] we studied bifurcation of homoclinic solutions of nonautonomous differential equations parametrized by a circle in terms of asymptotic behavior of its coefficients. The main result of that paper was obtained by means of an elementary index theorem relating the asymptotic stable bundles at plus and minus infinity to the index bundle of the family of Fredholm operators obtained by linearization of the equation at points of the trivial branch. In this paper we will discuss a refinement of this result in the special case of Hamiltonian systems but, this time, using an index theorem for one parameter families of real self-adjoint Fredholm operators.

On the analytical side a special feature of Hamiltonian systems is the variational structure of the induced nonlinear operator on function spaces. Thanks to this, bifurcation of homoclinics can be reduced to the study of bifurcation of critical points of a parametrized family of functionals. For functionals having only critical points with finite Morse indices it is a folklore result that bifurcation from a branch of critical points is produced by a jump in Morse index. Since the functionals under consideration here have infinite Morse indices, in order to detect bifurcation we have to consider instead the spectral flow of the family of self-adjoint Fredholm operators obtained by linearizing the equation at points of the trivial branch [5]. The spectral flow counts algebraically the number of eigenvalues crossing 0 , and hence it provides an appropriate substitute for the difference in Morse index in our setting.

On the topological side the peculiarity of the Hamiltonian case is due to the fact that the stable and unstable bundles of a linear Hamiltonian system are Lagrangian

Received by the editors February 9, 2007.

2000 Mathematics Subject Classification. Primary 37J45, 58E07; Secondary 34C37, 58J30, $53 \mathrm{D} 12$.

Key words and phrases. Bifurcation, Hamiltonian systems, homoclinic trajectories, spectral flow, Maslov index. 
with respect to the canonical symplectic structure on the phase space. In this context there is a well known obstruction to transversal intersection of two Lagrangian subbundles, namely the Maslov index. Denoting by $E^{s \pm}, E^{u \pm}$ the stable and unstable asymptotic bundles of the system at $\pm \infty$, respectively, our index theorem, Theorem 2.1 asserts that the spectral flow of the linearization at points of the trivial branch of stationary solutions equals the relative Maslov index $\mu\left(E^{s+}, E^{u-}\right)$. This, together with the bifurcation theorem in [5], leads to our main result, Theorem 2.2 (i): bifurcation of homoclinics arises whenever $\mu\left(E^{s+}, E^{u-}\right) \neq 0$.

It is easy to see that mod 2 reduction of the Maslov index coincides with the invariant $\omega_{1}$ used in [6]. Hence, Theorem 2.2 improves the main result of [6] in the case of Hamiltonian systems. However here we do not obtain a homoclinic solution $u$ with $\|u\|=\epsilon$ for all $\epsilon$ small enough, as was done in [6]. Instead, thanks to the variational structure of the problem, we obtain an estimate from below for the number of bifurcation points in terms of $\mu\left(E^{s+}, E^{u-}\right)$. This estimate, valid only in the Hamiltonian case, is of considerable interest since $\mu\left(E^{s+}, E^{u-}\right)$ is computable directly from the coefficients of the linearized system. In this regard Theorem 2.2 (ii) says, roughly speaking, the following: the larger the relative twist of coefficients of the linearized system at $\pm \infty$, the more homoclinic solutions bifurcate from the stationary branch.

Let us point out here that one can expect to find a nonvanishing bifurcation invariant computable from the coefficients of the linearization only in the presence of a topologically nontrivial parameter space such as $S^{1}$. Bifurcation invariants of a system parametrized by an interval depend on stable and unstable subspaces of the original equation. These subspaces can be found only by solving explicitly the corresponding nonautonomous differential equation (see [4]).

While the statements of the results here parallel those in [6, with an exception made in the estimate of Theorem 2.2 (ii), the proofs are completely different and use in an essential way both the symplectic and the variational structures of the problem. The index theorem is proved by means of a sequence of deformations of the principal symbol typical of elliptic topology 2 . The proof of the existence of bifurcation uses weak solutions in order to recast the problem to a general bifurcation theorem for critical points proved in 5 .

In [3] the bifurcation of intersections of Lagrangian submanifolds was studied by more geometric methods. The methods of that paper are, at least in principle, suitable to our purpose since homoclinic solutions of Hamiltonian systems correspond to intersection points of local stable and unstable manifolds of the vector field, which are Lagrangian in our case. However this approach runs short in technical problems connected with the realization of Lagrangian submanifolds of the cotangent bundle as the set of Lagrange multipliers of a family of smooth functionals.

\section{The Main Results}

We consider the system

$$
\left\{\begin{array}{l}
J u^{\prime}(t)+\nabla H(\lambda, t, u(t))=0 \\
\lim _{t \rightarrow+\infty} u(t)=\lim _{t \rightarrow-\infty} u(t)=0
\end{array}\right.
$$

depending on a parameter $\lambda$ belonging to the circle $S^{1}$.

Here $J$ is the matrix representing the canonical symplectic form $\omega$ on $\mathrm{R}^{2 n}$ against the scalar product, $H: S^{1} \times \mathrm{R} \times \mathrm{R}^{2 n} \rightarrow \mathrm{R}$ is $C^{2}$ with $\nabla H(\lambda, t, 0)=0$. 
Because of the last condition, for every $\lambda \in S^{1}$, the pair $(\lambda, 0)$ is a trivial stationary solution of the above equation. Homoclinic solutions are the nontrivial solutions of (2.1), i.e., solutions $(\lambda, u)$ with $u \neq 0$. The image of $u$ is a homoclinic trajectory of the corresponding Hamiltonian vector field.

For each $\lambda \in S^{1}$ the linearization of (2.1) at $u \equiv 0$ is given by

$$
\left\{\begin{array}{l}
J u^{\prime}(t)+A(\lambda, t) u(t)=0 \\
\lim _{t \rightarrow+\infty} u(t)=\lim _{t \rightarrow-\infty} u(t)=0
\end{array}\right.
$$

where $A(\lambda, t)=D_{u}^{2} H(\lambda, t, 0)$.

We will assume:

A0) $H(\lambda, t, u)=1 / 2\langle A(\lambda, t) u, u\rangle+G(\lambda, t, u)$, where $G(\lambda, t, u)$ vanishes up to second order at $u=0$ and there is a $\beta>0, C \geq 0$ and $g \in H^{1}(\mathrm{R})$ such that $\left|D_{u}^{2} G(\lambda, t, u)\right| \leq g(t)+C|u|^{\beta}$.

A1) As $t \rightarrow \pm \infty, A(\lambda, t)$ converges uniformly to a family of matrices $A(\lambda, \pm \infty)$ such that $S^{ \pm}(\lambda)=J A(\lambda, \pm \infty)$ are hyperbolic matrices, i.e., with no eigenvalues on the imaginary axis.

A2) For some fixed $\lambda_{0} \in S^{1}$, (2.2) admits only the trivial solution $u \equiv 0$.

It is shown in [7, Theorem 2.11, that, under assumption (A1), for each $\lambda \in S^{1}$ the operator $L_{\lambda}$ defined by

$$
L_{\lambda} u=J u^{\prime}(t)+A(\lambda, t) u(t)
$$

is an unbounded Fredholm self-adjoint operator in $L^{2}=L^{2}\left(\mathrm{R} ; \mathrm{R}^{2 n}\right)$ with domain $\mathcal{D}$ given by the space $H^{1}=H^{1}\left(\mathrm{R} ; \mathrm{R}^{2 n}\right)$ of all absolutely continuous $L^{2}$ functions with derivative in $L^{2}$.

The assumption (A1) can be reformulated as follows: if we define the principal symbol of $L_{\lambda}$ by $\sigma^{ \pm}\left(L_{\lambda}, \eta\right)=i J \eta+A^{ \pm}(\lambda, \pm \infty) \eta$, then the hyperbolicity of $S^{ \pm}(\lambda)$ is equivalent to the classical ellipticity condition $\operatorname{det} \sigma^{ \pm}\left(L_{\lambda}, \eta\right) \neq 0$ for all real $\eta \neq 0$. From this point of view systems of first order ordinary differential operators verifying (A1) should be called elliptic. Notice that here, since we are working on an unbounded domain and since the principal symbol should be invariant under compact perturbations, we must incorporate in the principal symbol the asymptotic coefficients of the lower order term at infinity.

In what follows instead of $\sigma^{ \pm}\left(L_{\lambda}, \eta\right)$ we will work directly with $\left(S^{+}(\lambda), S^{-}(\lambda)\right)$. A matrix of the form $M=J A$ with $A$ symmetric is called Hamiltonian. In terms of the symplectic form $\omega, M$ is Hamiltonian if and only if $\omega(M u, v)+\omega(u, M v)=0$. Thus, Hamiltonian matrices are elements of the Lie algebra of the group $S p(n)$ of symplectic isomorphisms of $\mathrm{R}^{2 n}$. Let us denote by $\mathcal{H}(2 n)$ the set of all Hamiltonian hyperbolic $2 n \times 2 n$-matrices. By assumption (A1), $S^{ \pm}(\lambda) \in \mathcal{H}(2 n)$ for all $\lambda \in S^{1}$. The map $S: S^{1} \rightarrow \mathcal{H}(2 n) \times \mathcal{H}(2 n)$ defined by $S(\lambda)=\left(S^{+}(\lambda), S^{-}(\lambda)\right)$ will be called the asymptotic symbol or simply the symbol of the family $L \equiv\left\{L_{\lambda}\right\}_{\lambda \in S^{1}}$.

To each closed path $L$ of unbounded self-adjoint Fredholm operators there is associated an integral valued invariant $\operatorname{sf}(L)$ called spectral flow. Roughly speaking, $\operatorname{sf}(L)$ is the number of negative eigenvalues of $L_{\lambda}$ that become positive as the parameter moves around the circle minus the number of positive eigenvalues that become negative. One possible interpretation parallels that of parity used in [6]. One can see the spectral flow as an intersection number of the path with the one 
codimensional singular variety $\Sigma$ of all noninvertible self-adjoint Fredholm operators. The variety $\Sigma$ is naturally co-oriented in the self-adjoint case. Therefore the intersection index is an integer rather than an integer mod 2 as in 6].

The construction of the spectral flow can be made in great generality, but here we will deal only with paths of operators defined by (2.3). We will use the approach of 77. Each $L_{\lambda}$ is a bounded perturbation of $D=J u^{\prime}$ and hence, endowing $H^{1}$ with its own norm, i.e., the graph norm of $D$, each $L_{\lambda}$ is bounded from $H^{1}$ to $L^{2}$ and, under our assumptions, $L: S^{1} \rightarrow \mathcal{L}\left(H^{1}, L^{2}\right)$ is a closed path in this space.

In what follows we will need to consider more general paths, which are not necessarily closed. If $L:[a, b] \rightarrow \mathcal{L}\left(H^{1}, L^{2}\right)$ is a $C^{1}$-path of Fredholm self-adjoint operators given by (2.3), the crossing form of $L$ at $\lambda \in[a, b]$ is the quadratic form $Q(L, \lambda)$ defined on $\operatorname{ker} L_{\lambda}$ by $Q(L, \lambda) v=\left\langle\dot{L}_{\lambda} v, v\right\rangle$. The point $\lambda$ is called a regular crossing point if $Q(L, \lambda)$ is a nondegenerate quadratic form. If all points of the singular set $\Sigma(L)=\left\{\lambda / \operatorname{ker} L_{\lambda} \neq 0\right\}$ are regular, then $\Sigma$ is a finite set and by definition $\operatorname{sf}(L)=\sum_{\lambda \in \Sigma} \operatorname{sig} Q(L, \lambda)$, where sig denotes the signature of a quadratic form. The spectral flow for general continuous paths is defined by approximation with differentiable paths having only regular crossing points. We will consider only nonclosed paths such that both $L_{a}$ and $L_{b}$ are invertible, because in this case the spectral flow is invariant by homotopies keeping the end points of the path invertible. Let us observe here that, on the contrary, the spectral flow of closed paths is invariant under general (free) homotopies [5. Clearly, the spectral flow is additive under concatenation of paths and a point-wise direct sum of operators.

If $A, \tilde{A}: S^{1} \times \mathrm{R} \rightarrow M_{\text {sym }}(2 n)$ are two families of symmetric matrices verifying (A1) and such that $\lim _{t \rightarrow \pm \infty} A(\lambda, t)=\lim _{t \rightarrow \pm \infty} \tilde{A}(\lambda, t)$, then

$$
H(\lambda, s) u=J u^{\prime}(t)+s A(\lambda, t) u(t)+(1-s) \tilde{A}(\lambda, t)
$$

is a homotopy of Fredholm self-adjoint operators between the associated differential operators $L$ and $\tilde{L}$. This shows that the spectral flow of $L$ depends only on the symbol of the family.

On the topological side, from assumption (A1) it follows that the families of vector spaces $E_{\lambda}^{s \pm}, E_{\lambda}^{u \pm}, \lambda \in S^{1}$, whose elements are (real) generalized eigenvectors corresponding to the spectrum of $S^{ \pm}(\lambda)=J A(\lambda, \pm \infty)$ on the left and right half plane, respectively, form two pairs of vector bundles $E^{s \pm}$ and $E^{u \pm}$ over $S^{1}$ such that each pair decomposes the trivial bundle $\Theta(2 n)=S^{1} \times \mathrm{R}^{2 n}$ into a direct sum $E^{s \pm} \oplus E^{u \pm}=\Theta(2 n)$. We will call them the stable and unstable asymptotic bundles, respectively.

Because the system (2.2) is Hamiltonian, the asymptotic stable and unstable bundles are Lagrangian subbundles of the symplectic bundle $\Theta(2 n)=S^{1} \times \mathrm{R}^{2 n}$ where $\mathrm{R}^{2 n}$ is endowed with the canonical symplectic form $\omega[u, v]=\langle J u, v\rangle$. In order to show this, it is enough to observe that both bundles can be characterized in terms of the decay of solutions of the linear system associated to $S^{ \pm}(\lambda)$. Indeed, $E_{\lambda}^{s \pm}=$ $\left\{v \mid \lim _{t \rightarrow+\infty} e^{t S^{ \pm}(\lambda)} v=0\right\}$ and similarly for $E_{\lambda}^{u \pm}$. Since $S^{ \pm}(\lambda)$ is Hamiltonian, the flow $e^{t S^{ \pm}(\lambda)}$ preserves the symplectic form $\omega$. Hence, for any $v \in E_{\lambda}^{s \pm}, \omega(v, v)=$ $\lim _{t \rightarrow \infty} \omega\left(e^{t S^{ \pm}(\lambda)} v, e^{t S^{ \pm}(\lambda)} v\right)=0$ and similarly with $E^{u \pm}$ when $t \rightarrow-\infty$. Thus $E^{s \pm}$ and $E^{u \pm}$ are isotropic, and since $E^{s \pm} \oplus E^{u \pm}=\Theta(2 n)$, they must be Lagrangian as well.

Let $\Lambda(n)$ be the space of all Lagrangian subspaces of $\mathrm{R}^{2 n}$ endowed with the topology induced by the metric $d\left(l, l^{\prime}\right)=\left\|P_{l}-P_{l^{\prime}}\right\|$, where $P_{l}$ denotes the orthogonal 
projector on the subspace $l$, and let us denote with $\mathcal{L}(n)$ the set of all Lagrangian subbundles of $\Theta(2 n)$. The Gauss map $\gamma_{E}$ of a Lagrangian subbundle $E$ of $\Theta(2 n)$, which assigns to each $\lambda \in S^{1}$ the fiber $E_{\lambda} \in \Lambda(n)$ of $E$, allows us to identify elements of $\mathcal{L}(n)$ with closed paths in $\gamma: S^{1} \rightarrow \Lambda(n)$. We will use this identification in order to associate to each Lagrangian subbundle its Maslov index.

Under the natural isomorphism $\mathrm{R}^{2 n} \simeq \mathrm{C}^{n}, J$ coincides with multiplication by $i$ and hence an orthogonal basis of a Lagrangian subspace $L$ gives rise to a unitary basis for $\mathrm{C}^{n}$. The choice of any such basis of $L$ leads to a unitary transformation sending $\mathrm{R}_{0}^{n}=\mathrm{R}^{n} \times\{0\}$ into $L$. Thus $U(n)$ acts transitively on $\Lambda_{n}$, the isotropy subgroup of $\mathrm{R}_{0}^{n}$ being clearly the orthogonal group $O(n)$. Therefore $\Lambda(n) \simeq U(n) / O(n)$ is a homogenous space and, since the determinant of an orthogonal matrix is \pm 1 , the map $\operatorname{det}^{2}: U(n) \rightarrow S^{1}$ factors through an analytic map $\rho: \Lambda(n) \rightarrow S^{1}$.

The Maslov index $\mu(\gamma) \in \mathrm{Z}$ of a closed path $\gamma: S^{1} \rightarrow \Lambda(n)$ is the winding number of the map $\rho \circ \gamma: S^{1} \rightarrow S^{1}$ [1]. The Maslov index $\mu(E)$ of a Lagrangian subbundle $E$ of $S$ is, by definition, the Maslov index of its Gauss map $\gamma_{E}$. Since the symplectic group is connected, it follows from the homotopy invariance of the winding number that $\mu(E)$ is invariant under the natural action of the symplectic group on $\mathcal{L}(n)$. In particular, $\mu(J E)=\mu(E)$.

Now, if $E \in \mathcal{L}(n)$ and $E^{\prime}$ is transverse to $E$, i.e., $E_{\lambda} \cap E_{\lambda}^{\prime}=0$ for all $\lambda \in S^{1}$, then, given any $\lambda$ and $x \in J E_{\lambda}$ there exists a unique $T_{\lambda} x \in E_{\lambda}$ such that $x+T_{\lambda} x \in E_{\lambda}^{\prime}$, i.e., $E^{\prime}$ is the graph of a vector bundle morphism $V: J E \rightarrow E$. It is easy to see that $E^{\prime}$ is Lagrangian if and only if $J T$ is a symmetric endomorphism of $J E$.

The deformation $\gamma:[0,1] \times S^{1} \rightarrow \Lambda(n)$ defined by

$$
\gamma(\lambda, t)=\operatorname{Graph} t T_{\lambda}
$$

is a homotopy between $\gamma_{E^{\prime}}$ and $\gamma_{J E}$. From this and the previous remark we obtain $\mu\left(E^{\prime}\right)=\mu(E)$ whenever $E^{\prime}$ is transverse to $E$.

In particular the Maslov indices of the asymptotic stable and unstable bundles $E^{s \pm \infty}$ and $E^{u \pm \infty}$ coincide.

Another important consequence of the above is that the difference $\mu(E)-\mu\left(E^{\prime}\right)$ can be considered as an obstruction to the deformation of the pair to a pair of transverse Lagragian subbundles.

The difference $\mu\left(E, E^{\prime}\right) \equiv \mu(E)-\mu\left(E^{\prime}\right)$ will be called the relative Maslov index of the pair. It enjoys better invariance properties than the ordinary Maslov index. By the previous discussion the latter is invariant under constant symplectic isomorphisms of the trivial bundle $\Theta(2 n)$. But $\mu\left(E, E^{\prime}\right)$ is invariant under general symplectic isomorphisms. Indeed, any symplectic isomorphism $\Psi$ of $\Theta(2 n)$ is of the form $\Psi(\lambda, u)=\left(\lambda, \bar{\Psi}_{\lambda} u\right)$ where $\bar{\Psi}$ is closed path in $S p(n)$. By the very definition of the Maslov index, $\mu(\Psi E)=\mu(E)+2$ win $(\operatorname{det} \bar{\Psi})$, where win denotes the winding number. Thus, if $E, E^{\prime}$ are two Lagrangian subbundles of $\Theta(2 n)$ and $\Psi$ is a symplectic automorphism of $\Theta(2 n)$, then $\mu\left(\Psi E, \Psi E^{\prime}\right)=\mu\left(E, E^{\prime}\right)$.

Since any symplectic bundle over $S^{1}$ is symplectically isomorphic to $\Theta(2 n)$, the relative Maslov index can be defined for pairs of Lagrangian subbundles of any symplectic vector bundle over $S^{1}$.

Summing up the previous discussion, each family $A: S^{1} \times \mathrm{R} \rightarrow M_{\text {sym }}(2 n)$ verifying assumption (A1) gives rise to a family $L: S^{1} \rightarrow \mathcal{L}\left(H^{1} ; L^{2}\right)$ of unbounded self-adjoint Fredholm operators defined by (2.3). To the family $L$ we can associate an analytical index given by the spectral flow $\operatorname{sf}(L)$ which counts the number of eigenvalues crossing 0 , and a topological index given by $\mu\left(E^{s+}, E^{u-}\right)$ which is 
a measure of the relative twist of the asymptotic bundles at infinity. With this understood, we have the following index theorem:

Theorem 2.1. If $L: S^{1} \rightarrow \mathcal{L}\left(H^{1} ; L^{2}\right)$ is as above, then $\operatorname{sf}(L)=\mu\left(E^{s+}, E^{u-}\right)$.

We will use this result in order to obtain a criterion for bifurcation of homoclinic solutions of the nonlinear equation (2.1) in terms of the asymptotic behavior of coefficients of the linearization.

Let $C_{0}^{1}\left(\mathrm{R} ; \mathrm{R}^{2 n}\right)$ be the space of all continuously differentiable $\mathrm{R}^{2 n}$ valued functions $u$, such that both $u$ and $u^{\prime}$ vanish at infinity. We endow $C_{0}^{1}=C_{0}^{1}\left(\mathrm{R} ; \mathrm{R}^{2 n}\right)$ with the norm ||$u||=\sup |u|+\sup \left|u^{\prime}\right|$.

A point $\lambda_{*} \in S^{1}$ is a bifurcation point for homoclinic solutions from the stationary branch if every neighborhood of $\left(\lambda_{*}, 0\right)$ in $S^{1} \times C_{0}^{1}$ contains a nontrivial solution $(\lambda, u)$ of (2.1).

Theorem 2.2. If the Hamiltonian $H$ satisfies (A1), (A2) and (A3), then

(i) There exists at least one bifurcation point $\lambda_{*} \in S^{1}$ for homoclinic solutions of (2.1) provided that $\mu\left(E^{s+}, E^{u-}\right) \neq 0$.

(ii) If moreover the singular set $\Sigma(L)$ is finite (e.g., if $L$ is either analytic or $L$ has only regular crossing points), then there are at least $\left|\mu\left(E^{s+}, E^{u-}\right)\right| / n$ bifurcation points.

\section{Proof of Theorem 2.1}

First of all we observe that any continuous map $S: S^{1} \rightarrow \mathcal{H}(2 n) \times \mathcal{H}(2 n)$ is the asymptotic symbol of some family of self-adjoint differential operators of type (2.3). Indeed, if $a(t)$ is any continuous function which vanishes identically for large negative $t$ and such that $a(t) \equiv 1$ for large positive $t$, then

$$
A(\lambda, t)=-a(t) J S^{+}(\lambda)-(1-a(t)) J S^{-}(\lambda)
$$

verifies (A1) and the symbol of the family of differential operators $L$ associated to $A$ is $S$. The homotopy (2.4) used in the previous section shows that $\operatorname{sf}(L)$ depends only on $S$ and, as a matter of fact, only on the homotopy class of this map. On the other hand, by definition of the topology on $\Lambda(n)$ the maps sending an element of $\mathcal{H}(2 n)$ into its stable and unstable subspaces is continuous. Thus, any homotopy between two symbols $S$ and $S^{\prime}$ induces a homotopy between the Gauss maps of $E^{s / u}(S)$ and $E^{s / u}\left(S^{\prime}\right)$, and hence also $\mu\left(E^{s+}, E^{u-}\right)$ depends only on the homotopy class of $S$.

Let $\pi\left[S^{1} ; \mathcal{H}(2 n) \times \mathcal{H}(2 n)\right]$ be the set of all free homotopy classes of maps from $S^{1}$ into $\mathcal{H}(2 n) \times \mathcal{H}(2 n)$. We have defined two functions sf, $\mu: \pi\left[S^{1} ; \mathcal{H}(2 n) \times \mathcal{H}(2 n)\right] \rightarrow \mathrm{Z}$. Theorem 2.1 is equivalent to the assertion $\mathrm{sf}=\mu$.

In order to prove the above assertion we will proceed by steps trying to find in each homotopy class $[S] \in \pi\left[S^{1} ; \mathcal{H}(2 n) \times \mathcal{H}(2 n)\right]$ a symbol of particularly simple form for which the equality can be checked directly.

Let us first introduce some notation. If $E, F$ are transverse Lagrangian subbundles of $\Theta(2 n)$, the decomposition $E \oplus F=\Theta(2 n)$ gives rise to the vector bundle projector $P_{E}^{F}$ projecting onto $E$ along $F$ and the complementary projector $P_{F}^{E}=\mathrm{id}-P_{E}^{F}$. We will denote by $S_{E}^{F}$ the reflection $P_{E}^{F}-P_{F}^{E}=2 P_{E}^{F}-I d$. The orthogonal projector onto $E$ will be denoted by $P_{E}$, and $S_{E}$ will be the corresponding reflection. 
Step 1. Each homotopy class in $\pi\left[S^{1} ; \mathcal{H}(2 n) \times \mathcal{H}(2 n)\right]$ contains a symbol $S$ of the form $\left(S_{E^{+}}, S_{E^{-}}\right)$, where $E^{ \pm}$are two Lagrangian subbundles of $\Theta(2 n)$ and $S_{E^{ \pm}}$ are the corresponding reflections.

Proof. Let $S=\left(S^{+}, S^{-}\right)$be any symbol. If we take $E=E^{s+}$ and $F=E^{u+}$, then the reflection $S_{E}^{F}$ clearly belongs to $\mathcal{H}(2 n)$, and since the restrictions of $S^{+}$to $E$ (respectively to $F$ ) have only positive (respectively negative) eigenvalues, it follows that $S(s, \lambda)=(1-s) S^{+}+s S_{E}^{F}$ is a homotopy with values in $\mathcal{H}(2 n)$ between $S^{+}$ and $S_{E}^{F}$. On the other hand, if $\gamma(t, \lambda)$ is the deformation defined in (2.5), the family $S(t, \lambda)=2 P_{\gamma(t, \lambda)}-\mathrm{Id}$ is a homotopy between $S_{E}^{F}$ and $S_{E}^{J E}=S_{E}$. Since the same argument holds for $S^{-}$as well, the claim is proved.

Our next step consists of a reduction of the proof to the case of a symbol of even simpler form.

Step 2. Given any symbol $S=\left(S_{E^{+}} S_{E^{-}}\right)$as in Step 1 there exists another symbol $\bar{S}: S^{1} \rightarrow \mathcal{H}(4 n) \times \mathcal{H}(4 n), \quad \bar{S}=\left(S_{\bar{E}^{+}}, S_{\bar{E}^{-}}\right)$having the same spectral flow and Maslov index as $S$ but such that $\bar{E}^{-}=\Theta_{0}(2 n)$, where $\Theta_{0}(2 n)$ is the Lagrangian subbundle $S^{1} \times \mathrm{R}^{2 n} \times\{0\}$ of the symplectic bundle $\Theta(4 n)$.

Proof. Let us consider the symplectic vector space $\overline{\mathrm{R}}^{4 n}=\mathrm{R}^{2 n} \times \mathrm{R}^{2 n}$ endowed with the symplectic form $\Omega=\pi_{1}^{*}(\omega)+\pi_{2}^{*}(\omega)$ where $\pi_{i}, i=1,2$, are the projections on the first and second factors, respectively. The space $\overline{\mathrm{R}}^{4 n}$ is the direct sum of $\mathrm{R}^{2 n}$ with itself in the symplectic category and (while isomorphic) should not be confused with $\mathrm{R}^{4 n}$ endowed with the canonical form. As before we will denote by $\bar{\Theta}(4 n)$ the trivial bundle over $S^{1}$ with fiber $\overline{\mathrm{R}}^{4 n}$ and by $\bar{J}=J \oplus J$ the corresponding complex structure.

Let us consider the lagrangian subbundle $F^{-}=E^{-} \times\{0\} \times\{0\} \times J E^{-}$of $\bar{\Theta}(4 n)$. Since $E^{-} \oplus J E^{-}=\Theta(2 n)$ is trivial (as vector bundle), we can find an orthonormal frame $f_{1}(\lambda), \ldots, f_{2 n}(\lambda)$ in $\bar{\Theta}(4 n)$ generating $F_{\lambda}^{-}$for all $\lambda \in S^{1}$.

Let $\Phi: \Theta(4 n) \rightarrow \bar{\Theta}(4 n)$ be the unique orthogonal symplectic isomorphism sending the canonical basis $e_{1}, \ldots, e_{2 n}$ of $R_{0}^{2 n}$ to $f_{1}(\lambda), \ldots, f_{2 n}(\lambda)$ and $J e_{1}, \ldots, J e_{2 n}$ to $\bar{J} f_{1}(\lambda), \ldots, \bar{J} f_{2 n}(\lambda)$. We take $\bar{F}^{+}=E^{+} \times\{0\} \times\{0\} \times J E^{-}$and define $\bar{E}^{ \pm}=$ $\Phi^{-1}\left(F^{ \pm}\right)$. We claim that the symbol $\bar{S}=\left(S_{\bar{E}^{-}}, S_{\bar{E}^{+}}\right)$has all the desired properties.

Clearly $\bar{E}^{-} \equiv \Theta_{0}(2 n)$. Moreover, by the invariance of the relative Maslov index under symplectic isomorphisms and additivity

$$
\mu\left(\bar{E}_{0}^{+}, \bar{E}_{0}^{-}\right)=\mu\left(F^{+}, F^{-}\right)=\mu\left(E^{+}\right)+\mu\left(J E^{-}\right)-\mu\left(E^{-}\right)-\mu\left(J E^{-}\right)=\mu\left(E^{+}, E^{-}\right),
$$

which shows that the relative Maslov index of $S$ and $\bar{S}$ are the same.

In order to prove that $\operatorname{sf}(\bar{S})=\operatorname{sf}(S)$ we will first show that the spectral flow of $\bar{S}$ coincides with the spectral flow of the symbol $\tilde{S}=\left(S_{F^{+}}, S_{F^{-}}\right)$. For this, we notice that $S_{F^{ \pm}}=\Phi S_{\bar{E}^{ \pm}} \Phi^{-1}$. Moreover, the isomorphism $\Phi$, by its very definition, is unitary, i.e., $\bar{J} \Phi=\Phi J$. It follows easily from these two facts that if $\bar{L}$ is a family of differential operators with symbol $\bar{S}$ and if $M_{\lambda}: L^{2}\left(\mathrm{R} ; \mathrm{R}^{4 n}\right) \rightarrow L^{2}\left(\mathrm{R} ; \overline{\mathrm{R}}^{4 n}\right)$ is the multiplication operator defined by $M_{\lambda} u=\Phi_{\lambda} u$, then $\tilde{L}_{\lambda}=M_{\lambda} L_{\lambda} M_{\lambda}^{-1}, \lambda \in S^{1}$, is a family with symbol $\tilde{S}$. By the invariance of the spectral flow under cogredience, [4. Theorem 4.2], we get $\overline{\operatorname{sf}}[\bar{S}]=\operatorname{sf} \bar{L}=\operatorname{sf} \tilde{L}=\overline{\operatorname{sf}}[\tilde{S}]$.

It remains only to show that the spectral flow of $\tilde{S}$ coincides with that of $S$. Being $P_{F}^{ \pm}=P_{E^{ \pm}} \oplus P_{J E^{-}}, \tilde{S}^{ \pm}$splits into a direct sum $\tilde{S}^{ \pm}=S_{E^{ \pm}} \oplus S_{J E^{-}}$. Therefore, if $\tilde{A}(\lambda, t)$ is the extension of $-J \tilde{S}^{ \pm}$to the cylinder $S^{1} \times \mathrm{R}$ given by (3.1), then 
$\tilde{A}(\lambda, t)=A(\lambda, t) \oplus B(\lambda)$, where $A(\lambda, t)=-a(t) J S^{+}(\lambda)-(1-a(t)) J S^{-}(\lambda)$ and $B(\lambda)=-J S_{J E^{-}}$is independent of $t$. Denoting again by $\tilde{L}_{\lambda}$ the corresponding differential operator we have that $\tilde{L}=L \oplus L^{\prime}$ with $L_{\lambda}^{\prime}=J u^{\prime}+B(\lambda) u$. By additivity $\operatorname{sf}[\tilde{S}]=\operatorname{sf} \tilde{L}=\operatorname{sf} L+\operatorname{sf} L^{\prime}$. But a function $u \in H^{1}$ belongs to Ker $L_{\lambda}^{\prime}$ if and only if $\dot{u}-S_{J E^{-}}(\lambda) u=0 . S_{J E^{-}}(\lambda)$ being a hyperbolic matrix implies that $u=0$. Thus $L^{\prime}$ is a path of invertible operators and sf $L^{\prime}=0$. Using the previous discussion we finally obtain $\operatorname{sf}[\bar{S}]=\overline{\operatorname{sf}}[\tilde{S}]=\operatorname{sf} L=\operatorname{sf}[S]$, which is what we wanted to show.

In the last step using symbols given by Step 2 we will consider the spectral flow as a function defined on $\pi\left[S^{1} ; \Lambda(n)\right]$ or equivalently on the first homotopy group $\pi_{1} \Lambda(n)$. In fact, since $\Lambda(n)$ is a simple space, the free and pointed homotopy classes of maps with values in $\Lambda(n)$ coincide. Let $\Xi(n)=\left\{(l, v) \in \Lambda(n) \times \mathrm{R}^{2 n} / v \in l\right\}$ be the tautological bundle over $\Lambda(n)$ and let $\Theta_{0}(n)=S^{1} \times \mathrm{R}_{0}^{n}$, as before. If $[\gamma] \in \pi_{1} \Lambda(n)$ we define $\operatorname{sf}([\gamma])=\operatorname{sf}([S])$, where $S=\left(S_{\gamma^{*} \Xi(n)}, S_{\Theta_{0}(n)}\right)$ and $\gamma^{*}$ denotes the pullback of a vector bundle by $\gamma$. From the additivity of the spectral flow under concatenation of paths it follows easily that sf: $\pi_{1} \Lambda(n) \rightarrow \mathrm{Z}$ is a homomorphism. We will compare it with the homomorphism $\mu: \pi_{1} \Lambda(n) \rightarrow \mathrm{Z}$ induced by the Maslov index.

Step 3. The homomorphism sf coincides with $\mu$.

Proof. For $\lambda=e^{i \theta}, 0 \leq \theta \leq 2 \pi$, let $l_{\lambda} \in \Lambda(1)$ be the line in $\mathrm{R}^{2}$ generated by $(\cos \theta / 2, \sin \theta / 2)$. The Maslov index of the path $l: S^{1} \rightarrow \Lambda(1)$ is its winding number and hence $\mu([l])=1$. Since, by Lemma 3.1 of [1, $\mu$ is an isomorphism, it follows that $[l]$ is a generator of $\pi_{1} \Lambda(1)$. On the other hand the map $i: \Lambda(1) \rightarrow \Lambda(n)$ sending a line $l^{\prime}$ into $l^{\prime} \times R_{0}^{n-1}$ commutes with the map $\rho$ used in the definition of the Maslov index. This implies that $\mu([i \circ l])=\mu([l])=1$ and therefore $[i \circ l]$ is a generator of $\pi_{1} \Lambda(n)$.

Using $l^{*} \circ i^{*} \Xi(n)=l^{*}\left(\Xi(1) \oplus \Theta_{0}(n-1)\right)=\Xi(1) \oplus \Theta_{0}(n-1)$ and the product property of the spectral flow we obtain

$$
\begin{aligned}
& \operatorname{sf}\left([i \circ l)=\operatorname{sf}\left(l^{*} \circ i^{*} \Xi(n), \Theta_{0}(n)\right)=\operatorname{sf}\left(\Xi(1) \oplus \Theta_{0}(n-1), \Theta_{0}(n)\right)\right. \\
& =\operatorname{sf}\left(\Xi(1), \Theta_{0}(1)\right)+\operatorname{sf}\left(\Theta_{0}(n-1), \Theta_{0}(n-1)=\operatorname{sf}\left(\Xi(1), \Theta_{0}(1)\right)=\operatorname{sf}([l]) .\right.
\end{aligned}
$$

Therefore, in order to show that $\mu=\mathrm{sf}$ it is enough to check that $\operatorname{sf}[l]=1$.

For $\lambda=e^{i \theta}, 0 \leq \theta \leq 2 \pi$, let $L_{\lambda}$ be the differential operator associated to

$$
A(\lambda, t)=\left\{\begin{array}{ll}
-2 \pi^{-1} \arctan t J S_{\theta} & \text { if } t \geq 0 \\
-2 \pi^{-1} \arctan t J S_{0} & \text { if } t \leq 0
\end{array}, \quad \text { where } S_{\theta}=\left(\begin{array}{rr}
\cos \theta & \sin \theta \\
\sin \theta & -\cos \theta
\end{array}\right)\right.
$$

is the reflection with respect to the line $l_{\lambda}$ generated by $(\cos \theta / 2, \sin \theta / 2)$. The symbol of the family $L$ is $S=\left(S_{\Xi(1)}, S_{\Theta_{0}(1)}\right)$ and hence $\operatorname{sf}([l])=\operatorname{sf}(L)$. But, if $u \in H^{1}$ and $L_{\lambda} u=0$, then $u(0) \in l_{\lambda} \cap l_{0}$, because $l_{\lambda}$ is positively invariant under the flow of $u^{\prime}=J A(\lambda, t) u$ and $l_{0}$ is negatively invariant. Therefore ker $L_{\lambda} \neq 0$ only if $\lambda=1$, i.e., $\theta=0$. Moreover ker $L_{1}$ is generated by $u_{*}(t)=\left(\sqrt{1+t^{2}} \exp t \arctan t, 0\right)$ and by a direct computation we obtain $Q(L, 1)\left(u_{*}\right)>0$. This shows that $\operatorname{sf}(L)=$ $\operatorname{sf}([l])=1$ and proves the last claim.

Using all the information at hand, we can finally conclude the proof of our Theorem 2.1. Given a symbol $S=\left(S^{+}, S^{-}\right)$, using Step 1 and Step 2 we can find a Lagrangian subbundle $E$ of $\Theta(4 n)$ and a symbol $\bar{S}$ with $\bar{S}^{+}=S_{E}$ and $\bar{S}^{-}=S_{\Theta_{0}(2 n)}$ having the same spectral flow and relative Maslov index of $S$. But $E=\gamma^{*} \Xi(2 n)$, 
where $\gamma$ is the Gauss map of $E$ and therefore

$$
\operatorname{sf}(\bar{S})=\operatorname{sf}([\gamma])=\mu([\gamma])=\mu\left(\gamma^{*} \Xi(2 n), \Theta_{0}(2 n)\right)=\mu(\bar{S})
$$

by the last step and definition of the relative Maslov index. Hence $\operatorname{sf}(S)=\mu(S)$, and the theorem is proved.

\section{Proof of Theorem 2.2}

The main theorem of [5] relates bifurcation of critical points of a one parameter family of $C^{2}$ functionals to the spectral flow of the family of its Hessians along the trivial branch. Here instead we are dealing with a family of unbounded self-adjoint operators $L_{\lambda} u=J u^{\prime}+A(\lambda, t) u$ with domain $H^{1}$ perturbed by a nonlinear map $u \rightarrow \nabla_{u} G(\lambda, t, u(t))$, the Nemytskii operator associated to $\nabla_{u} G$. Following [4] we will recast the problem (2.1) to one of the above types using the interpolation space $H^{1 / 2}=H^{1 / 2}\left(\mathrm{R}, \mathrm{R}^{2 n}\right)$. It is well known (see Lemma 10.3 of [8]) that the space $H^{1 / 2}$ is the form domain of $L_{\lambda}$, which means that the form $b_{\lambda}(u, v)=\left\langle L_{\lambda} u, v\right\rangle$ extends to a bounded bilinear form on $H^{1 / 2}$. On the other hand $H^{1 / 2}$ can be continuously embedded into $L^{p}, p>2([8$, Lemma 10.4). Let us choose a fixed $p \geq 2+2 \beta$, where $\beta$ is as in $(\mathrm{A} 0)$, and define

$$
\psi(\lambda, u)=\frac{1}{2} b_{\lambda}(u, u)+\int_{-\infty}^{+\infty} G(\lambda, t, u(t)) d t .
$$

With this choice of $p, \mid G\left(\lambda, t,\left.u|\leq g(t)+C| u\right|^{p-2}\right.$ and since $g \in L^{\frac{p}{p-2}}$, by the embedding of $H^{1 / 2}$ in $L^{p}$ and Theorem 5.4 of [4], it follows that $\psi: S^{1} \times H^{1 / 2} \rightarrow \mathrm{R}$ is a $C^{2}$ functional and

$$
d_{u} \psi(\lambda, u)(v)=b_{\lambda}(u, v)+\int_{-\infty}^{+\infty}\left\langle\nabla_{u} G(\lambda, t, u(t)), v(t)\right\rangle d t
$$

Moreover, for all $\lambda \in S^{1}$, the Hessian bilinear form at the critical point 0 of $\psi_{\lambda}$ is given by $D^{2} \psi_{\lambda}(0)(u, v)=b_{\lambda}(u, v)$.

The pair $(\lambda, u) \in S^{1} \times H^{1 / 2}$ is said to be a weak solution of equation (2.1) provided that (4.2) holds for all $v \in H^{1 / 2}$. Thus $(\lambda, u)$ is a weak solution of (2.1) if and only if $u$ is a critical point of the functional $\psi_{\lambda}=\psi(\lambda,-)$.

We claim that, if $(\lambda, u)$ is a weak solution of (2.1), then in fact $u$ belongs to $C_{0}^{1}\left(\mathrm{R} \mathrm{R}^{2 n}\right)$ and $(\lambda, u)$ solves (2.1) in the classical sense. We will first show that $u \in H^{1}$. Using continuity of $b$ and the density of $H^{1}$ in $H^{1 / 2}$, we observe that $b_{\lambda}(v, w)=\left\langle L_{\lambda} v, w\right\rangle$, for all $v \in H^{1}, w \in H^{1 / 2}$. Let $u \in H^{1 / 2}$ be a critical point of $\psi$. By A0), $z(t)=\nabla_{u} G(t, u(t))$ belongs to $L^{2}$. From the above relation it follows that $\left\langle u, L_{\lambda} v\right\rangle=-\langle z, v\rangle$ for all $v \in H^{1}$. $L_{\lambda}$ being self-adjoint implies that $u$ belongs to $H^{1}$ and $L_{\lambda} u=z$ almost everywhere. On the other hand, since any function of $H^{1}$ is continuous and vanishing at infinity, from the above equation we obtain that $u^{\prime}=-J\left[A(\lambda, t) u+\nabla_{u} G(t, u(t))\right] \in C_{0}$ and therefore $u \in C_{0}^{1}$ is a classical solution vanishing at infinity together with its derivative.

For definiteness let us assume that the point $\lambda_{0}=1$ in (A3). Taking $\lambda=e^{i \theta}$ we consider the path of $C^{2}$-functionals parametrized by the interval $[0,2 \pi], \psi\left(e^{i \theta},-\right)$ defined on $H^{1 / 2}$ by (4.1). By the Riesz theorem there is a unique bounded selfadjoint operator $T_{\lambda}$ such that $b_{\lambda}(u, v)=\left\langle T_{\lambda}, v\right\rangle_{H^{1 / 2}}$. Then $T_{e^{i \theta}}$ is the (operator) Hessian of $\psi\left(e^{i \theta},-\right)$ at the critical point 0 . 
By regularity $\operatorname{ker} L_{\lambda}=\operatorname{ker} T_{\lambda}$ for all $\lambda \in S^{1}$, and in particular for $\lambda=1$ we have that $T_{1}$ is nonsingular by (A3). Therefore the spectral flow (in the sense of [5]) of the path $T_{e^{i \theta}}$ on $[0,2 \pi]$ is well defined. We will use the regularity of solutions again in order to show that $\operatorname{sf}(L)=\operatorname{sf}(T)$.

Indeed, $\operatorname{ker} L_{\lambda}=\operatorname{ker} T_{\lambda}$, and if $L$ is $C^{1}$ and has only regular crossing points, then at each one of them $\left\langle\dot{L}_{\lambda} u, u\right\rangle=\left\langle\dot{T}_{\lambda} u, u\right\rangle_{H^{1 / 2}}$, for $u \in \operatorname{ker} L_{\lambda}$, by the representation formula. Hence, the crossing forms at any regular crossing point coincide and $\operatorname{sf}(L)=\operatorname{sf}(T)$ in this case. The general case is proved by approximation with regular paths via Theorem 4.22 of $[7]$.

Now, if $\mu\left(E^{s+}, E^{u-}\right) \neq 0$, then using Theorem 2.1 and applying Theorem 1 of $[5$ to the path $\psi\left(e^{i \theta},-\right)$ we obtain that every neighborhood $U$ of $S^{1} \times\{0\}$ in $S^{1} \times H^{1 / 2}$ contains a pair $(\lambda, u)$ with $u \neq 0$ a critical point of $\psi_{\lambda}$. Using compactness of $S^{1}$ we can find a sequence $\left(\lambda_{n}, u_{n}\right)$ of points as above such that $\lambda_{n} \rightarrow \lambda_{*}$ for some $\lambda_{*} \in S^{1}$ and $u_{n} \rightarrow 0$ in $H^{1 / 2}$. By regularity $u_{n} \in C_{0}^{1}$ and using the bootstrap again $u_{n} \rightarrow 0$ in that space. This proves (i).

In order to establish (ii) let us take a finite collection $\left\{J_{i}\right\}_{1 \leq i \leq k}$ of pairwise disjoint closed intervals centered at each singular point $\lambda_{i} \in \Sigma$. Since $T_{\lambda}$ is invertible for $\lambda \notin \bigcup_{i=1}^{k} J_{i}^{\circ}$, sf $T=\sum_{i=1}^{k} \operatorname{sf}\left(T, J_{i}\right)$. Arguing as in the proof of Theorem 4.1 of [5] we can choose $J_{i}$ small enough such that the spectral flow $\operatorname{sf}\left(T, J_{i}\right)$ equals the spectral flow of a path of symmetric operators on $\operatorname{ker} T_{\lambda_{i}}$.

We claim that $\operatorname{dim} \operatorname{ker} T_{\lambda}=\operatorname{dim} \operatorname{ker} L_{\lambda} \leq n$. Indeed, if $W_{\lambda}^{s}$ is the subspace of $\mathrm{R}^{2 n}$ whose elements are the initial values of solutions of $u^{\prime}=J A(\lambda, t) u$ decaying to 0 at plus infinity, then $W_{\lambda}^{s}$ is a Lagrangian subspace of $\mathrm{R}^{2 n}$ by the very same argument showing that asymptotic bundles are Lagrangian. In particular, $\operatorname{dim} W_{\lambda}^{s}=n$. Since the homomorphism $u \rightsquigarrow u(0)$ injects $\operatorname{ker} L_{\lambda}$ into $W_{\lambda}^{s}$, the claim is proved.

On the other hand the spectral flow of a path of symmetric endomorphisms of a finite dimensional Hilbert space is the difference of Morse indices at the end points, and hence its absolute value cannot be larger than the dimension of the space. Thus, we get $\left|\operatorname{sf}\left(T, J_{i}\right)\right| \leq n$ for $1 \leq i \leq k$. It follows then that the $\operatorname{sum} \sum_{i=1}^{k} \operatorname{sf}\left(T, J_{i}\right)$, being equal to $\mu\left(E^{s+}, E^{u-}\right)$, must contain at least $m=\left|\mu\left(E^{s+}, E^{u-}\right)\right| / n$ nonvanishing terms, which gives at least $m$ bifurcation points for critical points $\psi_{\lambda}$ and a fortiori at least $m$ bifurcation points for homoclinic trajectories.

\section{REFERENCES}

[1] V.I. Arnol'd, On a characteristic class entering into quantization conditions, Funk. Anal. i Priložen. 1 (1967), 1-13. MR0211415 (35:2296)

[2] M.F. Atiyah, V.K. Patodi, I.M. Singer, Spectral asymmetry and Riemannian geometry III, Math. Proc. Cambridge Philos. Soc. 79, no. 1 (1976), 71-99. MR0397799 (53:1655c)

[3] E. Ciriza, J. Pejsachowicz, A bifurcation theorem for Lagrangian intersections, Recent Trends in Nonlinear Analysis, Festschrift Dedicated to Alfonso Vignoli, Progress in Nonlinear Differential Equations and Their Applications 40, Birkhäuser, Basel, 2000, pp. 105 - 115. MR.1763139 (2001b:53101)

[4] P.M. Fitzpatrick, J. Pejsachowicz, C. A. Stuart, Spectral Flow for Paths of Unbounded Operators and Bifurcation of Critical Points, in preparation.

[5] P.M. Fitzpatrick, J. Pejsachowicz, L. Recht, Spectral flow and bifurcation of critical points of strongly indefinite functionals. Part I - General theory. J. Funct. Anal. 162 (1999), 52-95. MR.1674534(2000b:58021)

[6] J. Pejsachowicz, Bifurcation of homoclinics, Proc. Amer. Math. Soc. 136 (2008), 111-118. 
[7] J. Robbin, D. Salamon, The spectral flow and the Maslov index, Bull. London Math. Soc. 27 (1995), 1-33. MR1331677 (96d:58021)

[8] C.A. Stuart, Bifurcation into spectral gaps, Bull. Belg. Math. Soc. Simon Stevin, 1995, suppl., 1-59. MR.1361485 (96m:47115)

Dipartimento di Matematica, Politecnico di Torino, Corso Duca degli Abruzzi 24, 10129 TORINO, ITALY

E-mail address: jacobo.pejsachowicz@polito.it 